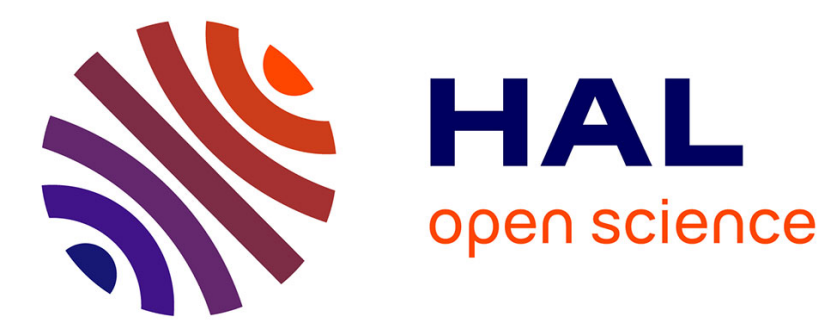

\title{
An Ergodic Description of Ground States
}

Eduardo Garibaldi, Philippe Thieullen

\section{To cite this version:}

Eduardo Garibaldi, Philippe Thieullen. An Ergodic Description of Ground States. Journal of Statistical Physics, 2015, 158, pp.359 - 371. 10.1007/s10955-014-1139-z . hal-01213721

\section{HAL Id: hal-01213721 \\ https://hal.science/hal-01213721}

Submitted on 31 Oct 2015

HAL is a multi-disciplinary open access archive for the deposit and dissemination of scientific research documents, whether they are published or not. The documents may come from teaching and research institutions in France or abroad, or from public or private research centers.
L'archive ouverte pluridisciplinaire HAL, est destinée au dépôt et à la diffusion de documents scientifiques de niveau recherche, publiés ou non, émanant des établissements d'enseignement et de recherche français ou étrangers, des laboratoires publics ou privés. 


\section{An ergodic description of ground states}

\author{
Eduardo Garibaldi* \\ Departamento de Matemática \\ Universidade Estadual de Campinas \\ 13083-859 Campinas - SP, Brasil \\ garibaldi@ime.unicamp.br
}

\author{
Philippe Thieullen ${ }^{\dagger}$ \\ Institut de Mathématiques \\ Université Bordeaux 1, CNRS, UMR 5251 \\ F-33405 Talence, France \\ Philippe.Thieullen@math.u-bordeaux1.fr
}

October 5, 2010

Abstract

\section{Introduction}

\section{$2 \quad$ Framework and Main Results}

Given a finite spin set $\mathrm{F}$, we introduce the configuration space $\Omega=\mathrm{F}^{\mathbb{Z}^{d}}$. Its elements $\boldsymbol{\omega} \in \Omega$ are written as $\boldsymbol{\omega}=\left\{\omega_{j}\right\}_{j \in \mathbb{Z}^{d}}$. Endowed with the product topology of discrete topologies, $\Omega$ is a compact metrizable space. Indeed, for $j=\left(j_{1}, \ldots, j_{d}\right) \in \mathbb{Z}^{d}$ one may consider the $L^{1}$ norm $\|j\|=\left|j_{1}\right|+\ldots+\left|j_{d}\right|$ and define then a metric on $\Omega$ by

$$
\mathrm{d}(\boldsymbol{\omega}, \overline{\boldsymbol{\omega}})=2^{-\inf \left\{\|j\|: \omega_{j} \neq \bar{\omega}_{j}\right\}}
$$

which is compatible with the product topology.

Notice that $\mathbb{Z}^{d}$ acts on $\Omega$ by translation. Let $\left\{e_{1}, \ldots, e_{d}\right\}$ be the canonical basis for the lattice $\mathbb{Z}^{d}$. For each $i \in\{1, \ldots, d\}$, we consider the shift transformation $\theta_{i}: \Omega \rightarrow \Omega$ given by $\theta_{i}(\boldsymbol{\omega})=\left\{\omega_{j+e_{i}}\right\}_{j \in \mathbb{Z}^{d}}$. Given $j=\left(j_{1}, \ldots, j_{d}\right) \in \mathbb{Z}^{d}$, we define $\theta^{j}:=\theta_{1}^{j_{1}} \circ \theta_{2}^{j_{2}} \circ \cdots \circ \theta_{d}^{j_{d}}$.

Let $\mathcal{F}$ denote the collection of finite subsets of $\mathbb{Z}^{d}$. The diameter and the boundary of $A \in \mathcal{F}$ are respectively $\operatorname{diam}(A)=\max \{\|j-k\|: j, k \in A\}$ and $\partial A:=\left\{j \in \mathbb{Z}^{d} \backslash A:\|j-k\|=1\right.$ for some $\left.k \in A\right\}$. For $A \in \mathcal{F}$ and $\boldsymbol{\omega} \in \Omega$, we denote the restriction $\left.\boldsymbol{\omega}\right|_{A}$ simply by $\boldsymbol{\omega}_{A}$.

Definition 2.1. By an invariant interaction family we mean any collection of continuous maps $\Phi_{A}: \Omega \rightarrow \mathbb{R}$, indexed by $A \in \mathcal{F}$, such that

1. $\Phi_{j+A}(\boldsymbol{\omega})=\Phi_{A}\left(\theta^{j}(\boldsymbol{\omega})\right)$, for all $j \in \mathbb{Z}^{d}$ and $\boldsymbol{\omega} \in \Omega$;

*supported by FAPESP 2009/17075-8

${ }^{\dagger}$ supported by ANR BLANC07-3_187245, Hamilton-Jacobi and Weak KAM Theory 
2. $\boldsymbol{\omega}_{A}=\overline{\boldsymbol{\omega}}_{A}$ implies $\Phi_{A}(\boldsymbol{\omega})=\Phi_{A}(\overline{\boldsymbol{\omega}})$.

We say that $\left\{\Phi_{A}\right\}_{A \in \mathcal{F}}$ is an invariant short range interaction family if in addition there exists an integer $r>0$ such that $\Phi_{A} \equiv 0$ whenever diam $(A)>r$. In this case, we say also that the invariant interaction family has range $r>0$. For $r=1$, one in particular says to have an interaction of nearest neighbors.

Given an invariant interaction family $\left\{\Phi_{A}\right\}_{A \in \mathcal{F}}$, we introduce the associated hamiltonian $H: \mathcal{F} \times \Omega \rightarrow \mathbb{R}$ defined by

$$
H(\Lambda, \boldsymbol{\omega})=H_{\Lambda}(\boldsymbol{\omega}):=\sum_{A: A \cap \Lambda \neq \emptyset} \Phi_{A}(\boldsymbol{\omega}), \quad \forall \Lambda \in \mathcal{F}, \boldsymbol{\omega} \in \Omega .
$$

Notice also that the hamiltonian inherits the invariance of the interaction family, namely, $H_{\Lambda} \circ \theta^{j}=H_{j+\Lambda}$ for any $j \in \mathbb{Z}^{d}$.

Remark 2.2. We say that $\left\{\Phi_{A}\right\}_{A \in \mathcal{F}}$ is an invariant long range interaction family if $\Phi_{A} \not \equiv 0$ for sets $A$ with arbitrarily large diameter. In this case, one shall assume a summability condition:

$$
\sum_{0 \in A}\left\|\Phi_{A}\right\|_{\infty}<\infty
$$

which is obviously trivial for the short range situation. Notice that the associated hamiltonian is then well defined, since

$\left\|H_{\Lambda}\right\|_{\infty} \leq \sum_{j \in \Lambda} \sum_{j \in A}\left\|\Phi_{A}\right\|_{\infty}=\sum_{j \in \Lambda} \sum_{0 \in A}\left\|\Phi_{A} \circ \theta^{j}\right\|_{\infty}=\# \Lambda \sum_{0 \in A}\left\|\Phi_{A}\right\|_{\infty}<\infty, \quad \forall \Lambda \in \mathcal{F}$.

For $\boldsymbol{\omega}, \overline{\boldsymbol{\omega}} \in \Omega$ and $\Lambda \in \mathcal{F}$, we denote by $\overline{\boldsymbol{\omega}}_{\Lambda} \boldsymbol{\omega}_{\mathbb{Z}^{d} \backslash \Lambda}$ the configuration of $\Omega$ that coincides with $\overline{\boldsymbol{\omega}}$ on $\Lambda$ and with $\boldsymbol{\omega}$ on $\mathbb{Z}^{d} \backslash \Lambda$. We also introduce the following notation

$$
\Lambda_{n+1}:=\{-n,-n+1, \ldots, 0, \ldots, n-1, n\}^{d}, \quad \forall n \in \mathbb{N} .
$$

Definition 2.3. We say that $\boldsymbol{\omega} \in \Omega$ is a minimizing configuration with respect to the hamiltonian $H$ if

$$
H_{\Lambda}(\boldsymbol{\omega}) \leq H_{\Lambda}\left(\overline{\boldsymbol{\omega}}_{\Lambda} \boldsymbol{\omega}_{\mathbb{Z}^{d} \backslash \Lambda}\right), \quad \forall \overline{\boldsymbol{\omega}} \in \Omega, \Lambda \in \mathcal{F} .
$$

We define the minimizing ergodic value of the hamiltonian $H$ as the constant

$$
\bar{H}:=\inf _{\boldsymbol{\omega} \in \Omega} \liminf _{n \rightarrow \infty} \frac{1}{\# \Lambda_{n}} H_{\Lambda_{n}}(\boldsymbol{\omega}) .
$$

Our main result concerning these minimizing objects is the following one.

Theorem 2.4. Let $H$ be a hamiltonian defined by an invariant short range interaction family. The set of minimizing configurations with respect to the hamiltonian $H$ is a non-empty invariant closed set. For a minimizing configuration $\boldsymbol{\omega} \in \Omega$, the limit $\lim _{n \rightarrow \infty} \frac{1}{\# \Lambda_{n}} H_{\Lambda_{n}}(\boldsymbol{\omega})$ always exists. Moreover, there are minimizing configurations $\boldsymbol{\omega} \in \Omega$ for which

$$
\lim _{n \rightarrow \infty} \frac{1}{\# \Lambda_{n}} H_{\Lambda_{n}}(\boldsymbol{\omega})=\bar{H}
$$




\section{Proof of Theorem 2.4}

The proof of Theorem 2.4 follows from a series of results regrouped in this section.

Proposition 3.1. Let $H$ be a hamiltonian defined by an invariant short range interaction family. There exist minimizing configurations for the hamiltonian $H$. The set of minimizing configurations with respect to $H$ is invariant and closed.

Proof. For each $\Lambda \in \mathcal{F}$, denote

$$
\Omega_{\Lambda}:=\left\{\boldsymbol{\omega} \in \Omega: H_{\Lambda}(\boldsymbol{\omega}) \leq H_{\Lambda}(\overline{\boldsymbol{\omega}}), \forall \overline{\boldsymbol{\omega}} \in \Omega \text { with } \overline{\boldsymbol{\omega}}_{\mathbb{Z}^{d} \backslash \Lambda}=\boldsymbol{\omega}_{\mathbb{Z}^{d} \backslash \Lambda}\right\} .
$$

Notice that $\Omega_{\Lambda}$ is non-empty: for any fixed configuration $\boldsymbol{\omega} \in \Omega$, it clearly contains the minimum points of the map $\overline{\boldsymbol{\omega}}_{\Lambda} \in \mathrm{F}^{\Lambda} \mapsto H_{\Lambda}\left(\overline{\boldsymbol{\omega}}_{\Lambda} \boldsymbol{\omega}_{\mathbb{Z}^{d} \backslash \Lambda}\right) \in \mathbb{R}$. Besides, each $\Omega_{\Lambda}$ is a closed set, since the map $\boldsymbol{\omega} \in \Omega \mapsto H_{\Lambda}\left(\overline{\boldsymbol{\omega}}_{\Lambda} \boldsymbol{\omega}_{\mathbb{Z}^{d} \backslash \Lambda}\right)-H_{\Lambda}(\boldsymbol{\omega}) \in \mathbb{R}$ is continuous (actually, locally constant) for all $\overline{\boldsymbol{\omega}}_{\Lambda} \in \mathrm{F}^{\Lambda}$.

The family $\left\{\Omega_{\Lambda}\right\}_{\Lambda \in \mathcal{F}}$ is monotone: $\Lambda \subset \Lambda^{\prime} \Rightarrow \Omega_{\Lambda^{\prime}} \subset \Omega_{\Lambda}$. Indeed, suppose that $\boldsymbol{\omega}^{\prime} \in \Omega_{\Lambda^{\prime}}$. Let $\overline{\boldsymbol{\omega}} \in \Omega$ be such that $\overline{\boldsymbol{\omega}}_{\mathbb{Z}^{d} \backslash \Lambda}=\boldsymbol{\omega}_{\mathbb{Z}^{d} \backslash \Lambda}^{\prime}$. As $\Lambda \subset \Lambda^{\prime}$, we get $\overline{\boldsymbol{\omega}}_{\mathbb{Z}^{d} \backslash \Lambda^{\prime}}=\boldsymbol{\omega}_{\mathbb{Z}^{d} \backslash \Lambda^{\prime}}^{\prime}$. Hence, $H_{\Lambda^{\prime}}\left(\boldsymbol{\omega}^{\prime}\right) \leq H_{\Lambda^{\prime}}(\overline{\boldsymbol{\omega}})$. Since

$$
H_{\Lambda^{\prime}}=H_{\Lambda}+\sum_{\substack{A \cap \Lambda^{\prime} \neq \emptyset \\ A \cap \Lambda=\emptyset}} \Phi_{A}
$$

and $\Phi_{A}\left(\boldsymbol{\omega}^{\prime}\right)=\Phi_{A}(\overline{\boldsymbol{\omega}})$ whenever $\emptyset \neq A \cap \Lambda^{\prime} \subset \Lambda^{\prime} \backslash \Lambda$, we clearly obtain that $H_{\Lambda}\left(\boldsymbol{\omega}^{\prime}\right) \leq H_{\Lambda}(\overline{\boldsymbol{\omega}})$, which means that $\boldsymbol{\omega}^{\prime} \in \Omega_{\Lambda}$.

Therefore, the family of closed sets $\left\{\Omega_{\Lambda}\right\}_{\Lambda \in \mathcal{F}}$ has the finite intersection property: $\bigcap_{i=1}^{N} \Omega_{\Lambda_{i}} \supset \Omega_{\bigcup_{i=1}^{N} \Lambda_{i}} \neq \emptyset$. By compactness, there exists $\omega \in \Omega_{\Lambda}$ for all $\Lambda \in \mathcal{F}$.

The set of minimizing configurations with respect to $H$ is exactly $\bigcap_{\Lambda \in \mathcal{F}} \Omega_{\Lambda}$, which is clearly closed. Besides, since $\theta^{-j}\left(\Omega_{\Lambda}\right)=\Omega_{j+\Lambda}$ for all $j \in \mathbb{Z}^{d}$, this set is also invariant.

Denote the Birkhoff sum by $S_{\Lambda} \Psi:=\sum_{j \in \Lambda} \Psi \circ \theta^{j}$.

Definition 3.2. Given an invariant short range interaction family $\left\{\Phi_{A}\right\}_{A \in \mathcal{F}}$, we introduce

$$
\Psi_{0}:=\sum_{A: 0 \in A} \frac{1}{\# A} \Phi_{A} .
$$

Notice that $\Psi_{0}$ is a real valued function since the above sum is actually finite. Moreover, we have the following property.

Lemma 3.3. The map $\Psi_{0}$ satisfies

$$
\sup _{\Lambda \in \mathcal{F}} \sup _{\boldsymbol{\omega}_{\Lambda}=\overline{\boldsymbol{\omega}}_{\Lambda}} \frac{1}{\# \partial \Lambda}\left(S_{\Lambda} \Psi_{0}(\boldsymbol{\omega})-S_{\Lambda} \Psi_{0}(\overline{\boldsymbol{\omega}})\right)<\infty .
$$

In order to prove this lemma, given $\Lambda \in \mathcal{F}$ and $r>0$, we define

$$
\operatorname{Int}_{r} \Lambda:=\{j \in \Lambda: d(j, \partial \Lambda) \geq r\} .
$$


Proof. Notice that

$$
\begin{aligned}
S_{\Lambda} \Psi_{0} & =\sum_{j \in \Lambda} \sum_{A: 0 \in A} \frac{1}{\# A} \Phi_{A} \circ \theta^{j}=\sum_{j \in \Lambda} \sum_{A: j \in A} \frac{1}{\# A} \Phi_{A} \\
& =\sum_{A: A \cap \Lambda \neq \emptyset} \sum_{j \in A \cap \Lambda} \frac{1}{\# A} \Phi_{A}=\sum_{A: A \cap \Lambda \neq \emptyset} \frac{\#(A \cap \Lambda)}{\# A} \Phi_{A} .
\end{aligned}
$$

Assume the invariant interaction family $\left\{\Phi_{A}\right\}_{A \in \mathcal{F}}$ has range $r>0$. Whenever $\boldsymbol{\omega}_{\Lambda}=\overline{\boldsymbol{\omega}}_{\Lambda}$, it is easy to see that

$$
\begin{aligned}
S_{\Lambda} \Psi_{0}(\boldsymbol{\omega})-S_{\Lambda} \Psi_{0}(\overline{\boldsymbol{\omega}}) & =\sum_{A \cap\left(\Lambda \backslash \operatorname{Int}_{r} \Lambda\right) \neq \emptyset} \frac{\#(A \cap \Lambda)}{\# A}\left(\Phi_{A}(\boldsymbol{\omega})-\Phi_{A}(\overline{\boldsymbol{\omega}})\right) \\
& \leq 2 \sum_{A \cap\left(\Lambda \backslash \operatorname{Int}_{r} \Lambda\right) \neq \emptyset}\left\|\Phi_{A}\right\|_{\infty} \leq 2 \#\left(\Lambda \backslash \operatorname{Int}_{r} \Lambda\right) \sum_{0 \in A}\left\|\Phi_{A}\right\|_{\infty},
\end{aligned}
$$

from which the statement follows immediately.

The relation of $\Psi_{0}$ with the associated hamiltonian is given below.

Proposition 3.4. Suppose $\left\{\Phi_{A}\right\}_{A \in \mathcal{F}}$ is an invariant interaction family with range $r>0$. If $H$ is the associated hamiltonian, then

$$
\left\|H_{\Lambda}-S_{\Lambda} \Psi_{0}\right\|_{\infty} \leq \#\left(\Lambda \backslash \operatorname{Int}_{r} \Lambda\right) \sum_{0 \in A}\left\|\Phi_{A}\right\|_{\infty}
$$

Proof. Using (3.1), we obtain

$$
H_{\Lambda}-S_{\Lambda} \Psi_{0}=\sum_{A \cap \operatorname{Int}_{r} \Lambda \neq \emptyset}\left[1-\frac{\#(A \cap \Lambda)}{\# A}\right] \Phi_{A}+\sum_{A \cap\left(\Lambda \backslash \operatorname{Int}_{r} \Lambda\right) \neq \emptyset}\left[1-\frac{\#(A \cap \Lambda)}{\# A}\right] \Phi_{A} .
$$

Notice that if $A \in \mathcal{F}$ has diameter less than $r$ and intersects $\operatorname{Int}_{r} \Lambda$, then $A \subset \Lambda$. Thus, the first term in the right side of the above equation is equal to zero. Hence, we get

$$
\left\|H_{\Lambda}-S_{\Lambda} \Psi_{0}\right\|_{\infty} \leq \sum_{A \cap\left(\Lambda \backslash \operatorname{Int}_{r} \Lambda\right) \neq \emptyset}\left\|\Phi_{A}\right\|_{\infty} \leq \#\left(\Lambda \backslash \operatorname{Int}_{r} \Lambda\right) \sum_{0 \in A}\left\|\Phi_{A}\right\|_{\infty} .
$$

We remark that, for $\Lambda_{n}=(-n, n)^{d} \cap \mathbb{Z}^{d}$, one has $\#\left(\Lambda_{n} \backslash \operatorname{Int}_{r} \Lambda_{n}\right) \leq C_{d} r^{d} n^{d-1}$, for some constant $C_{d}>0$ which depends just on the dimension $d$. In particular, we immediately obtain the following corollary.

Corollary 3.5. Let $H$ be a hamiltonian defined by an invariant short range interaction family. Then

$$
\bar{H}=\inf _{\boldsymbol{\omega} \in \Omega} \liminf _{n \rightarrow \infty} \frac{1}{\# \Lambda_{n}} S_{\Lambda_{n}} \Psi_{0}(\boldsymbol{\omega}) .
$$

We may now prove the second statement of Theorem 2.4. 
Proposition 3.6. Let $H$ be a hamiltonian defined by an invariant short range interaction family. Then, for any minimizing configuration $\boldsymbol{\omega}$ with respect to $H$, the limit

$$
\lim _{n \rightarrow \infty} \frac{1}{\# \Lambda_{n}} H_{\Lambda_{n}}(\boldsymbol{\omega})
$$

does exist.

Proof. Let $\boldsymbol{\omega}$ be a minimizing configuration for $H$. By Proposition 3.4, it is equiv-

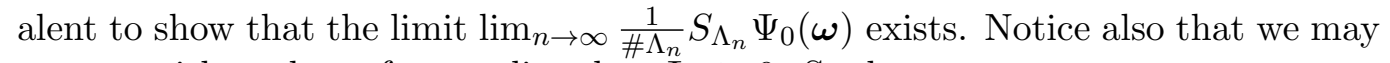
assume without loss of generality that $\Psi_{0} \geq 0$. So denote

$$
L=\liminf _{n \rightarrow \infty} \frac{1}{\# \Lambda_{n}} S_{\Lambda_{n}} \Psi_{0}(\boldsymbol{\omega})
$$

and, given $\epsilon>0$, consider a positive integer $N$ such that $\frac{1}{\# \Lambda_{N}} S_{\Lambda_{N}} \Psi_{0}(\boldsymbol{\omega})<L+\epsilon$.

Consider integers $m$ and $\ell$ with $m \geq 1$ and $\ell=0,1, \ldots, N-1$. Notice that, for suitable integers $j_{1}, \ldots, j_{m^{d}} \in \Lambda_{m N}$, we may write

$$
S_{\Lambda_{m N+\ell}} \Psi_{0}=\sum_{k=1}^{m^{d}} S_{\Lambda_{N}} \Psi_{0} \circ \theta^{j_{k}}+\sum_{j \in \Lambda_{m N+\ell} \backslash \Lambda_{m N}} \Psi_{0} \circ \theta^{j} .
$$

Define then $\boldsymbol{\omega}_{N, k}:=\left(\theta^{j_{k}}(\boldsymbol{\omega})\right)_{j_{k}+\Lambda_{N}} \boldsymbol{\omega}_{\mathbb{Z}^{d} \backslash\left(j_{k}+\Lambda_{N}\right)}$ and $\overline{\boldsymbol{\omega}}_{N, k}:=\theta^{-j_{k}}\left(\boldsymbol{\omega}_{N, k}\right)$. As $\boldsymbol{\omega}$ is a minimizing configuration, one thus gets

$$
H_{j_{k}+\Lambda_{N}}(\boldsymbol{\omega}) \leq H_{j_{k}+\Lambda_{N}}\left(\boldsymbol{\omega}_{N, k}\right)=H_{\Lambda_{N}}\left(\overline{\boldsymbol{\omega}}_{N, k}\right) .
$$

Recall that $\left\|S_{\Lambda_{N}} \Psi_{0} \circ \theta^{j}-H_{j+\Lambda_{N}}\right\|_{\infty} \leq \Gamma N^{d-1}$ for every $j \in \mathbb{Z}^{d}$, where $\Gamma=$ $\Gamma\left(d, r,\left\{\Phi_{A}\right\}\right)=C_{d} r^{d} \sum_{0 \in A}\left\|\Phi_{A}\right\|_{\infty}$. Therefore, we have that

$$
\begin{aligned}
S_{\Lambda_{m N+\ell}} \Psi_{0}(\boldsymbol{\omega}) & \leq \sum_{k=1}^{m^{d}} H_{\Lambda_{N}}\left(\overline{\boldsymbol{\omega}}_{N, k}\right)+m^{d} \Gamma N^{d-1}+\sum_{j \in \Lambda_{m N+\ell} \backslash \Lambda_{m N}} \Psi_{0} \circ \theta^{j}(\boldsymbol{\omega}) \\
& \leq \sum_{k=1}^{m^{d}} S_{\Lambda_{N}} \Psi_{0}\left(\overline{\boldsymbol{\omega}}_{N, k}\right)+2 m^{d} \Gamma N^{d-1}+2^{d}\left[(m+1)^{d}-m^{d}\right] N^{d}\left\|\Psi_{0}\right\|_{\infty} .
\end{aligned}
$$

Notice that $\left(\overline{\boldsymbol{\omega}}_{N, k}\right)_{\Lambda_{N}}=\boldsymbol{\omega}_{\Lambda_{N}}$. We use now Lemma 3.3 to guarantee that there exists a constant $\kappa>0$ such that $S_{\Lambda_{N}} \Psi_{0}\left(\overline{\boldsymbol{\omega}}_{N, k}\right)-S_{\Lambda_{N}} \Psi_{0}(\boldsymbol{\omega}) \leq \kappa \# \partial \Lambda_{N}$.

We obtain that

$$
\begin{aligned}
& S_{\Lambda_{m N+\ell}} \Psi_{0}(\boldsymbol{\omega}) \leq \\
& \quad \leq m^{d}\left[S_{\Lambda_{N}} \Psi_{0}(\boldsymbol{\omega})+\kappa \# \partial \Lambda_{N}+2 \Gamma N^{d-1}+2^{d}\left(\left(1+\frac{1}{m}\right)^{d}-1\right) N^{d}\left\|\Psi_{0}\right\|_{\infty}\right],
\end{aligned}
$$

which yields

$$
\begin{aligned}
& \frac{1}{\# \Lambda_{m N+\ell}} S_{\Lambda_{m N+\ell}} \Psi_{0}(\boldsymbol{\omega}) \leq \\
& \quad \leq \frac{1}{\# \Lambda_{N}} S_{\Lambda_{N}} \Psi_{0}(\boldsymbol{\omega})+\kappa \frac{\# \partial \Lambda_{N}}{\# \Lambda_{N}}+2 \frac{\Gamma}{N}+\left(\left(1+\frac{1}{m}\right)^{d}-1\right)\left\|\Psi_{0}\right\|_{\infty} .
\end{aligned}
$$


Hence, it follows that

$$
\limsup _{n \rightarrow \infty} \frac{1}{\# \Lambda_{n}} S_{\Lambda_{n}} \Psi_{0}(\boldsymbol{\omega}) \leq L+\epsilon+\kappa \frac{\# \partial \Lambda_{N}}{\# \Lambda_{N}}+2 \frac{\Gamma}{N} .
$$

Since the integer $N$ can be taken arbitrarily large and $\epsilon>0$ can be chosen as close as one wants to zero, the proof is complete.

We will adopt an ergodic point of view. To that end, denote by $\mathcal{M}$ the set of Borel probability measures, equipped with the weak* topology. We consider the compact convex subset of invariant probabilities

$$
\mathcal{M}_{\theta}:=\left\{\mu \in \mathcal{M}: \mu \circ \theta^{j}=\mu, \forall j \in \mathbb{Z}^{d}\right\} .
$$

We have then the following characterization of the minimizing ergodic value $\bar{H}$.

Proposition 3.7. Let $H$ be a hamiltonian defined by an invariant short range interaction family. Then

$$
\bar{H}=\min _{\mu \in \mathcal{M}_{\theta}} \int_{\Omega} \Psi_{0}(\boldsymbol{\omega}) d \mu(\boldsymbol{\omega}) .
$$

Proof. By the ergodic decomposition theorem (see, for example, Theorem 2.3.3 in [3]), one may suppose that $\mu \in \mathcal{M}_{\theta}$ is ergodic. Therefore, by Birkhoff's ergodic theorem (see, for instance, Theorem 2.1.5 in [3]), any configuration $\boldsymbol{\omega}$ belonging to the support of $\mu$ satisfies

$$
\lim _{n \rightarrow \infty} \frac{1}{\# \Lambda_{n}} S_{\Lambda_{n}} \Psi_{0}(\boldsymbol{\omega})=\int_{\Omega} \Psi_{0}(\boldsymbol{\omega}) d \mu(\boldsymbol{\omega}) .
$$

So thanks to Corollary 3.5, we have that $\bar{H} \leq \inf _{\mu \in \mathcal{M}_{\theta}} \int \Psi_{0} d \mu$.

For $\epsilon>0$, consider a configuration $\boldsymbol{\omega}_{\epsilon} \in \Omega$ and an arbitrarily large integer $n_{\epsilon}>0$ such that $\frac{1}{\# \Lambda_{n_{\epsilon}}} S_{\Lambda_{n_{\epsilon}}} \Psi_{0}\left(\boldsymbol{\omega}_{\epsilon}\right)<\bar{H}+\epsilon$ and define a Borel probability measure

$$
\mu_{\epsilon}:=\frac{1}{\# \Lambda_{n_{\epsilon}}} \sum_{j \in \Lambda_{n_{\epsilon}}} \delta_{\theta^{j}\left(\boldsymbol{\omega}_{\epsilon}\right)} \in \mathcal{M} .
$$

Let $\mu$ be any weak* accumulation probability for the family $\left\{\mu_{\epsilon}\right\}_{\epsilon>0}$ when $\epsilon$ goes to zero. Clearly by construction, $\int_{\Omega} \Psi_{0}(\boldsymbol{\omega}) d \mu(\boldsymbol{\omega}) \leq \bar{H}$. So in order to obtain the opposite inequality, it is enough to argue that $\mu$ is invariant. However, notice that, for every $i=1,2, \ldots, n$ and for all $f \in C^{0}(\Omega)$, one has

$$
\left|\int\left(f \circ \theta_{i}-f\right) d \mu_{\epsilon}\right| \leq \frac{1}{\# \Lambda_{n_{\epsilon}}} 2 \# \partial \Lambda_{n_{\epsilon}}\|f\|_{\infty} \rightarrow 0 \quad \text { as } \quad n_{\epsilon} \rightarrow \infty
$$

which indeed shows the invariance of $\mu$.

By a minimizing probability we mean an invariant probability $\mu$ that minimizes the average value $\int \Psi_{0} d \mu$, namely, such that $\bar{H}=\int \Psi_{0} d \mu$. Their existence is guaranteed by the previous proposition. Moreoveor, by the ergodic decomposition theorem, there always exist ergodic minimizing probabilities.

Recall now that a point at the support of an invariant probability is said to be generic if it belongs to a subset of full measure. We may complete the proof of Theorem 2.4 with the following result. 
Theorem 3.8. Suppose $\left\{\Phi_{A}\right\}_{A \in \mathcal{F}}$ is an invariant interaction family with range $r>0$. Then there are generic points $\boldsymbol{\omega}$ at the support of any ergodic minimizing probability which are minimizing configurations for the associated hamiltonian $H$ and satisfy

$$
\lim _{n \rightarrow \infty} \frac{1}{\# \Lambda_{n}} H_{\Lambda_{n}}(\boldsymbol{\omega})=\bar{H}
$$

Proof. For $W \in \mathrm{F}^{\Lambda_{M}}, M \in \mathbb{N}$, consider the characteristic function $\chi_{W}: \Omega \rightarrow\{0,1\}$ which has value 1 at a point $\overline{\boldsymbol{\omega}} \in \Omega$ if, and only if, $\overline{\boldsymbol{\omega}}_{\Lambda_{M}}=W$.

Let $\mu \in \mathcal{M}_{\theta}$ be an ergodic minimizing probability. Let us denote by $b(\phi)$ the subset of $\Omega$ of full measure for which Birkhoff's ergodic theorem holds with respect to the integrable map $\phi: \Omega \rightarrow \mathbb{R}$. Obviously, any point

$$
\boldsymbol{\omega} \in \operatorname{supp}(\mu) \cap b\left(\Psi_{0}\right) \cap \bigcap_{W \in \mathrm{F}^{\Lambda_{M}, M \in \mathbb{N}}} b\left(\chi_{W}\right)
$$

is generic and verify

$$
\lim _{n \rightarrow \infty} \frac{1}{\# \Lambda_{n}} H_{\Lambda_{n}}(\boldsymbol{\omega})=\lim _{n \rightarrow \infty} \frac{1}{\# \Lambda_{n}} S_{\Lambda_{n}} \Psi_{0}(\boldsymbol{\omega})=\int \Psi_{0} d \mu=\bar{H}
$$

Suppose on the contrary that $\boldsymbol{\omega}$ is not a minimizing configuration. Hence, there shall exist $\tilde{\boldsymbol{\omega}} \in \Omega, \tilde{N} \in \mathbb{N}$ and $\tilde{\eta}>0$ such that

$$
\tilde{\boldsymbol{\omega}}_{\mathbb{Z}^{d} \backslash \Lambda_{\tilde{N}}}=\boldsymbol{\omega}_{\mathbb{Z}^{d} \backslash \Lambda_{\tilde{N}}} \quad \text { and } \quad H_{\Lambda_{\tilde{N}}}(\tilde{\boldsymbol{\omega}})<H_{\Lambda_{\tilde{N}}}(\boldsymbol{\omega})-\tilde{\eta} .
$$

Since in particular $\boldsymbol{\omega} \in \bigcap_{M \in \mathbb{N}} b\left(\chi_{\boldsymbol{\omega}_{\Lambda_{M}}}\right)$, we have that

$$
\lim _{n \rightarrow \infty} \frac{1}{\# \Lambda_{n}} S_{\Lambda_{n}} \chi \boldsymbol{\omega}_{\Lambda_{\tilde{N}+r}}(\boldsymbol{\omega})=\mu\left(\bigcup_{\overline{\boldsymbol{\omega}} \in \Omega} \boldsymbol{\omega}_{\Lambda_{\tilde{N}+r}} \overline{\boldsymbol{\omega}}_{\mathbb{Z}^{d} \backslash \Lambda_{\tilde{N}+r}}\right)=: \lambda_{\tilde{N}+r}>0
$$

Therefore, for $n$ large enough, one guarantees that $S_{\Lambda_{n}} \chi_{\boldsymbol{\omega}_{\Lambda_{\tilde{N}+r}}}(\boldsymbol{\omega})>\# \Lambda_{n} \frac{{ }_{\lambda_{\tilde{N}+r}}}{2}$.

Denote $A_{n}:=\left\{j \in \Lambda_{n}: \boldsymbol{\omega}_{\Lambda_{\tilde{N}+r}+j}=\boldsymbol{\omega}_{\Lambda_{\tilde{N}+r}}\right\}$. Let then $B_{n} \subset A_{n}$ be a maximal subcollection of indices such that $\left(\Lambda_{\tilde{N}+r}+j\right) \cap\left(\Lambda_{\tilde{N}+r}+k\right)=\emptyset$ whenever $j, k \in B_{n}$. Since for all $j \in A_{n}$ there must exist $k \in B_{n}$ such that $\left(\Lambda_{\tilde{N}+r}+j\right) \cap\left(\Lambda_{\tilde{N}+r}\right) \neq \emptyset$, it follows that $S_{\Lambda_{n}} \chi_{\boldsymbol{\omega}_{\Lambda_{\tilde{N}+r}}}(\boldsymbol{\omega})=\# A_{n} \leq \# B_{n} \cdot \# \Lambda_{\tilde{N}+r}$, which yields for $n$ large enough

$$
\frac{1}{\# \Lambda_{n}} \# B_{n}>\frac{1}{\# \Lambda_{\tilde{N}+r}} \frac{\lambda_{\tilde{N}+r}}{2}
$$

Thus, for $n$ sufficiently large, let us introduce the configuration $\boldsymbol{\omega}^{n} \in \Omega$ as $\boldsymbol{\omega}_{\Lambda_{\tilde{N}}+j}^{n}=\tilde{\boldsymbol{\omega}}_{\Lambda_{\tilde{N}}}$ for all $j \in B_{n}$, and $\boldsymbol{\omega}_{\mathbb{Z}^{d} \backslash \sqcup_{j \in B_{n}}\left(\Lambda_{\tilde{N}}+j\right)}=\boldsymbol{\omega}_{\mathbb{Z}^{d} \backslash \sqcup_{j \in B_{n}}\left(\Lambda_{\tilde{N}}+j\right)}$. From the construction, one gets that $H_{\Lambda_{\tilde{N}}+j}\left(\boldsymbol{\omega}^{n}\right)=H_{\Lambda_{\tilde{N}}}(\tilde{\boldsymbol{\omega}})$ for each $j \in B_{n}$. Notice then 
that

$$
\begin{aligned}
H_{\Lambda_{n}}\left(\boldsymbol{\omega}^{n}\right) & =\sum_{j \in B_{n}} H_{\Lambda_{\tilde{N}}+j}\left(\boldsymbol{\omega}^{n}\right)+\sum_{\substack{A \cap \Lambda_{n} \neq \emptyset, A \cap \sqcup_{j \in B_{n}}\left(\Lambda_{\tilde{N}}+j\right)=\emptyset}} \Phi_{A}\left(\boldsymbol{\omega}^{n}\right) \\
& <\# B_{n}\left(H_{\Lambda_{\tilde{N}}}(\boldsymbol{\omega})-\tilde{\eta}\right)+\sum_{\substack{A \cap \Lambda_{n} \neq \emptyset, A \cap \sqcup_{j \in B_{n}}\left(\Lambda_{\tilde{N}}+j\right)=\emptyset}} \Phi_{A}(\boldsymbol{\omega}) \\
& =H_{\Lambda_{n}}(\boldsymbol{\omega})-\tilde{\eta} \# B_{n}+\sum_{j \in B_{n}}\left(H_{\Lambda_{\tilde{N}}}(\boldsymbol{\omega})-H_{\Lambda_{\tilde{N}}}\left(\theta^{j}(\boldsymbol{\omega})\right)\right) .
\end{aligned}
$$

By the very definition of $A_{n}$, one obtains that $H_{\Lambda_{\tilde{N}}}(\boldsymbol{\omega})=H_{\Lambda_{\tilde{N}}}\left(\theta^{j}(\boldsymbol{\omega})\right)$ for any $j \in B_{n}$. In this way, let us also assume that $n$ is large enough in order that

$$
\frac{1}{\# \Lambda_{n}} H_{\Lambda_{n}}\left(\boldsymbol{\omega}^{n}\right)<\bar{H}+\frac{1}{\# \Lambda_{\tilde{N}+r}} \frac{\lambda_{\tilde{N}+r}}{4} \tilde{\eta} .
$$

Hence, for $n$ sufficiently large, it is not difficult to see that

$$
\frac{1}{\# \Lambda_{n}} H_{\Lambda_{n}}\left(\boldsymbol{\omega}^{n}\right)<\bar{H}-\frac{1}{\# \Lambda_{\tilde{N}+r}} \frac{\lambda_{\tilde{N}+r}}{4} \tilde{\eta}
$$

which contradicts the definition (2.2) of the constant $\bar{H}$.

\section{References}

[1] A. Bovier, Statistical mechanics of disordered systems: A mathematical perspective, Cambridge Series in Statistical and Probabilistic Mathematics 18, Cambridge University Press, Cambridge, 2006.

[2] H. O. Georgii, Gibbs measures and phase transitions, De Gruyter Studies in Mathematics 9, Walter de Gruyter, Berlin, 1988.

[3] G. Keller, Equilibrium states in ergodic theory, London Mathematical Society Students Texts 42, Cambridge University Press, Cambridge, 1998. 